\title{
Purely Inseparable Extensions of Unique Factorization Domains
}

\author{
By \\ Jeffery LANG*
}

\begin{abstract}
§0. Introduction
This article attempts to set some further groundwork for the study of codimension-one cycles of purely inseparable coverings of varieties in characteristic $p$. Thus it represents, hopefully, a preliminary effort. The simplest type of purely inseparable cover of a variety $X$ with coordinate ring $A$ in characteristic $p \neq 0$ is obtained by taking $Y=\operatorname{Spec}\left(A\left[{ }^{p} \sqrt{g}\right]\right)$ for some $g \in A$. Efforts to relate the codimension one cycles of $X$ and $Y([8],[2])$ led to the ring-theoretic question,

I. If $A$ is a UFD of characteristic $p \neq 0$, for what $g \in A$ is $A\left[{ }^{p} \sqrt{g}\right]$ a UFD?

A natural place to begin to investigate (I) is with $A$ a polynomial ring defined over a field $k$ of characteristic $p>0$. When $k$ is perfect (I) can be restated,

II. For what $g \in A=k\left[x_{1}, \ldots, x_{n}\right]$ is $A^{p}[g]$ a UFD?

This paper investigates (I) in Section 5 when $A$ is the coordinate ring of a surface $X$ defined as a complete intersection and extends (II) in Section 3 to study the divisor classes of rings of the form $k\left[x_{1}^{p}, \ldots, x_{n}^{p}, g_{1}, \ldots, g_{n-1}\right]$.

After a few brief preliminaries in Section 1, some tools for calculating $C l(A)$ are developed in Section 2, that generalize ([8], (2.6)) and ([5], II(1.3)), which played an important role in showing that for a general choice of $g \in A$ $=k\left[x_{1}, \ldots, x_{n}\right] A^{p}[g]$ is factorial (See [5], II(2.6)). In Section 4 some examples are considered. The reader is also referred to two excellent references for the subject of divisor classes of Krull rings, Samuel's 1964 Tata notes [11] and Fossum's "The Divisor Class Group of a Krull Domain"[4].
\end{abstract}

Communicated by K. Saito, May 2, 1989. Revised November 13, 1989.

* Department of Mathematics, University of Kansas, Lawrence, KS 66045, U.S.A.

Partially supported by the General Research Fund at the University of Kansas \# 3818-XX-0038. 


\section{§1. Notation and Preliminaries}

(0.1) $k$-an algebraically closed field of characteristic $p \neq 0$.

(0.2) $A=k\left[x_{1}, \ldots, x_{n}\right]$-a polynomial ring in $n$-indeterminates over $k$.

(0.3) Given $f_{1}, \ldots, f_{n} \in A, J\left(f_{1}, \ldots, f_{n}\right)$ is the determinant of the matrix $\left[D_{i}\left(f_{j}\right)\right], 1 \leq i, j \leq n$, where $D_{i}=\frac{\partial}{\partial x_{i}}$.

(0.4) Given $h \in A$, let $\operatorname{deg}_{x_{1}}(h)$ denote the degree of $h$ in $x_{i}$, $\operatorname{deg}_{x_{i}, x_{j}}(h)$ denote the degree of $h$ in $x_{i}$ and $x_{j}$, etc..., let $\operatorname{deg}(h)$ denote the total degree of $h$.

(0.5) $A_{k}^{n}$ denotes the affine $n$-space over $k$.

(0.6) $f_{1}, \ldots, f_{n-1} \in A$ are said to satisfy condition $\left(^{*}\right)$ if the variety $V \subseteq A_{k}^{n}$ defined by the $n-1$ by $n-1$ minors of the matrix $\left[\begin{array}{ccc}D_{1}\left(f_{1}\right) & \cdots & D_{n}\left(f_{1}\right) \\ \vdots & & \vdots \\ D_{1}\left(f_{n-1}\right) & \cdots & D_{n}\left(f_{n-1}\right)\end{array}\right]$ has dimension less than $n-2$.

(0.7) If $R$ is a Krull ring denote by $C l(R)$ the divisor class group of $R([11]$, page 18) (cf. (0.2)).

(0.8) If $X$ is a noetherian integral separated scheme which is regular in codimension one, denote by $C l(X)$ the group of Weil divisors of $X([7]$, page 130).

(0.9) If $R$ is a noetherian integrally closed domain, then $R$ is a Krull ring and $X=\operatorname{Spec}(R)$ will be regular in codimension one, and $C l(R)$ and $C l(X)$ defined above are isomorphic.

(0.10) Given $g, f_{1}, \ldots, f_{n-2} \in A$, let $P$ be the ideal in $A$ generated by $f_{1}, \ldots, f_{n-2}$ and $B=A / P$. For $f \in A$, denote its image in $B$ by $\bar{f}$. Then we say that $g$, $f_{1}, \ldots, f_{n-2}$ satisfies condition $\left(^{* *}\right)$ if all three of the following conditions are satisfied.

(i) $P$ is a height $n-2$ prime ideal in $A$.

(ii) $\bar{g} \notin B^{p}=k\left[\bar{x}_{1}^{p}, \ldots, \bar{x}_{n}^{p}\right]$

(iii) The ring $A[\omega] /\left(\omega^{p}-g, f_{1}, \ldots, f_{n-2}\right)$ is regular in codimension one.

Note that the ring in (iii) is a domain by (i) and (ii) and it is regular in codimension one if and only if it is normal ([7], pg. 186, Proposition 8.23).

(0.11) For a prime number $p$, we will let $\mathbb{F}_{p}$ denote the finite field of order $p$ and the set of integers $\{0,1, \ldots, p-1\}$. It will be clear from the context which is meant.

1.1. Theorem. Let $A \subset B$ be Krull rings. Suppose that either $B$ is integral over $A$ or that $B$ is a flat A-algebra. Then there is a well defined group homomorphism $\varphi: C l(\mathrm{~A}) \rightarrow \operatorname{Cl}(B)$.

Let $B$ be a Krull ring of characteristic $p>0$. Let $\Delta$ be a derivation of $L$, the quotient field of $B$, such that $\Delta(B) \subset B$. Let $K=\operatorname{ker}(\Delta)$ and $A$ 
$=B \cap K$. Then $A$ is a Krull ring with $B$ integral over $A$. By (1.1) we have a map $\varphi: C l(A) \rightarrow C l(B)$ (see [11] pp. 19-20). Set $\mathscr{L}=\left\{t^{-1} \Delta t \in B \mid t \in L\right\}$ and $\mathscr{L}^{\prime}$ $=\left\{u^{-1} \Delta u \mid u\right.$ is a unit in $\left.\mathrm{B}\right\}$. Then $\mathscr{L}$ is an additive group with subgroup $\mathscr{L}^{\prime}$.

1.2. Theorem. (a) There exists a canonical monomorphism $\bar{\varphi}: \operatorname{ker} \varphi$ $\rightarrow \mathscr{L} / \mathscr{L}^{\prime}$. (b) If $[L: K]=p$ and $\Delta(B)$ is not contained in any height one prime of $B$, then $\bar{\varphi}$ is an isomorphism ([11], p. 62).

1.3. Theorem. (a) If $[L: K]=p$, then there exists $a \in A$ such that $\Delta^{p}=a \Delta$, (b) $t \in L$ is equal to $u^{-1} \Delta u$ for some $u \in L$ if and only if $\Delta^{p-1} t-a t+t^{p}$ $=0([11]$, pp. 63-64).

\section{§2. Computational Tools}

Let $k$ be an algebraically closed field of characteristic $p>0$. Let $A$ $=k\left[x_{1}, \ldots, x_{n}\right]$ be a polynomial ring in $n$-indeterminates over $k$. Let $f_{1}, \ldots, f_{n-1} \in A$. Define a derivation $D$ on $L=k\left(x_{1}, \ldots, x_{n}\right)$ by $D(h)$ $=J\left(h, f_{1}, \ldots, f_{n-1}\right)$ where $J$ represents the determinant of the Jacobian matrix. That is,

$$
J\left(h, f_{1}, \ldots, f_{n-1}\right)=\operatorname{det}\left[\begin{array}{cccc}
D_{1}(h) & D_{2}(h) & \cdots & D_{n}(h) \\
D_{1}\left(f_{1}\right) & D_{2}\left(f_{1}\right) & \cdots & D_{n}\left(f_{1}\right) \\
\vdots & \vdots & \vdots & \vdots \\
D_{1}\left(f_{n-1}\right) & D_{2}\left(f_{n-1}\right) & \cdots & D_{n}\left(f_{n-1}\right)
\end{array}\right] \text { where } D_{j}=\frac{\partial}{\partial x_{j}}
$$

We have the following generalization of $([8]$, page $395,(2.6))$. We let $A^{\prime}$ $=k\left[x_{1}^{p}, \ldots, x_{n}^{p}, f_{1}, \ldots, f_{n-1}\right]$.

2.1. Proposition. Assume $D \neq 0$. Then (i) there exists $a \in A$ such that $D(a)=0$ and $D^{p}=a D$, (ii) $a$ is given by $a=(-1)^{n} \sum_{j=1}^{n-1} \sum_{r_{j}=0}^{p-1} f_{1}^{r_{1}} \cdots f_{n-1}^{r_{n-1}} \nabla\left(f_{1}^{p-r_{1}-1} \ldots\right.$ $\left.f_{n-1}^{p-r_{n-1}-1}\right)$, (iii) For all $t \in L, D^{p-1} t-a t=(-1)^{n-1} \sum_{j=1}^{n-1} \sum_{r_{j}=0}^{p-1} f_{1}^{r_{1}} \cdots f_{n-1}^{r_{n-1}} \nabla\left(f_{1}^{p-r_{1}-1}\right.$ $\left.\cdots f_{n-1}^{p-r_{n-1}-1} t\right)$, where $\nabla=\left(D_{1}^{p-1} \cdots D_{n}^{p-1}\right)$.

Proof (i) For each $i=1, \ldots, n-1, f_{i} \notin k\left(x_{1}^{p}, \ldots, x_{n}^{p}, f_{1}, \ldots, f_{i-1}\right)$ (where we let $\left.f_{0}=0\right)$ since $D \neq 0$. It then follows that

2.1.1. $\left[L: L^{\prime}\right]=p$ and $L^{\prime}=D^{-1}(0)$, where $L^{\prime}$ is the quotient field of $A^{\prime}$.

By (1.3) there exists $a \in A \cap L^{\prime}$ such that $D^{p}=a D$.

(ii) Will follow from (iii) by letting $t=1$.

(iii) $\operatorname{Case}(\mathrm{I})$ : The $f_{i}$ contain no monomials that are $p$-th powers and the $f_{i}$ 
satisfy condition $(*)$.

For each $i=1, \ldots, n-1$, let $\Delta_{i}$ be the derivation on $L$ defined by

$$
\Delta_{i}(h)=\operatorname{det}\left[\begin{array}{ccc}
D_{1}(h) & \cdots & D_{n-1}(h) \\
D_{1}\left(f_{1}\right) & \cdots & D_{n-1}\left(f_{1}\right) \\
\vdots & & \vdots \\
D_{1}\left(f_{i-1}\right) & \cdots & D_{n-1}\left(f_{i-1}\right) \\
D_{1}\left(f_{i+1}\right) & \cdots & D_{n-1}\left(f_{i+1}\right) \\
\vdots & & \vdots \\
D_{1}\left(f_{n-1}\right) & \cdots & D_{n-1}\left(f_{n-1}\right)
\end{array}\right]
$$

Since $D \neq 0$, we may assume after a permutation of the $x_{i}$ that $\Delta_{i}\left(f_{i}\right) \neq 0$ for each $i$. Now for each $1 \leq i \leq n-1$, let $E_{i}=\left(1 / \Delta_{i}\left(f_{i}\right)\right) \cdot \Delta_{i}$ and $E$ $=E_{1}^{p-1} \cdots E_{n-1}^{p-1}$.

2.1.2. Claim: $E\left(D^{p-1} t-a t\right)=\nabla t$, for all $t \in L$.

Proof of (2.1.2). If $t \in A, \operatorname{deg}(D t) \leq M+\operatorname{deg} t-n$, where $M=\sum_{i=1}^{n-1} \operatorname{deg} f_{i}$. It then follows that $\operatorname{deg}(a) \leq(p-1)(M-n)$ and

2.1.3. $\operatorname{deg}\left(D^{p-1} t-a t\right) \leq \operatorname{deg}(t)+(p-1)(M-n)$, for all $t \in A$.

Given $h \in A^{\prime}$, there is a unique $\beta_{\bar{r}} \in A$, for each $\bar{r} \in \mathbb{F}_{p}^{n-1}$, such that $h$ $=\sum_{\bar{r}} \beta_{\bar{r}} f^{\bar{r}}$ (where for $\left.\bar{r}=\left(r_{1}, \ldots, r_{n-1}\right) \in \mathbb{F}_{p}^{n-1}, f^{\bar{r}}=f_{1}^{r_{1}} \cdots f_{n-1}^{r_{n-1}}\right)$. We have that for each $i=1, \ldots, n-1, E_{i}(h)=\sum_{\bar{r}} r_{i} \beta \frac{p}{r} f^{\vec{r}}$, where $\bar{r}^{\prime}=\left(r_{1}, \ldots, r_{i}-1, \ldots, r_{n-1}\right)$. Then $E_{i}(h) \in A^{\prime}$ and $\operatorname{deg}\left(E_{i}(h)\right) \leq \operatorname{deg}(h)-\operatorname{deg}\left(f_{i}\right)$. Given $t \in A, D^{p-1} t-a t \in A^{\prime}$ by (3.2) below, the proof of which is independent of this section, since $D^{p}=a D$ and $D a=0$. By (2.1.3) we have for all $t \in A$,

$$
E\left(D^{p-1} t-a t\right)=0 \text {, or }
$$

2.1.4

$$
\operatorname{deg}\left(E\left(D^{p-1} t-a t\right)\right) \leq \operatorname{deg} t-(p-1) n .
$$

Any differential operator on $k\left(x_{1}, \ldots, x_{n}\right)$ can be written uniquely as a linear combination of $D_{1}^{s_{1}} \cdots D_{n}^{s_{n}}, 0 \leq s_{i} \leq p-1$, with coefficients in $L$. Thus there exists unique $\alpha_{\bar{r}} \in L$, for each $\bar{r} \in \mathbb{F}_{p}^{n}$ such that

2.1.5. $E\left(D^{p-1}-a I\right)=\sum_{\bar{r}} \alpha_{\bar{r}} \partial^{\bar{r}}$, where for $\bar{r}=\left(r_{1}, \ldots, r_{n}\right), \partial^{\bar{r}}=D_{1}^{r_{1}} D_{2}^{r_{2}} \cdots D_{n}^{r_{n}}$.

Proceed by induction on $\sum r_{i}$ to show $\alpha_{\bar{r}}=0$ for $\bar{r} \neq(p-1, \ldots, p-1)$. By (2.1.4), $E\left(D^{p-1}(1)-a(1)\right)=0$. By $(2.1 .5) \alpha_{(0, \ldots, 0)}=0$. Assume $\alpha_{\bar{r}}=0$ for all $\bar{r}$ 
with $\sum_{r_{i}<k}<n(p-1)$. Let $\bar{r}^{*}=\left(r_{1}^{*}, \ldots, r_{n}^{*}\right)$ be such that $\sum r_{i}^{*}=k$. Substitute $t=x_{1}^{r_{1}^{*}} \cdots x_{n}^{r_{n}^{*}}$ into (2.1.5) and use (2.1.4) to obtain $r_{1}^{*} ! \cdots r_{n}^{*} ! \alpha_{r^{*}}=0$ which implies $\alpha_{r^{*}}=0$. Therefore

$$
E\left(D^{p-1}-a I\right)=\alpha \nabla, \text { for some } \alpha \in L .
$$

Apply both sides of (2.1.6) to $\left(x_{1} \cdots x_{n}\right)^{p-1}$ and use (2.1.4) to see that $\alpha \in k$.

To compute $\alpha$, we first note that $\alpha$ in (2.1.6) is invariant under a linear change of variables. This may be checked by one coordinate change at a time. If $x_{1}$ say, is replaced by $\alpha_{1} x_{1}+\cdots+\alpha_{n} x_{n}+\alpha_{n+1}\left(\alpha_{i} \in k\right)$ then $D_{1}$ becomes $\alpha_{1} D_{1}$ and $\nabla$ becomes $\alpha_{1}^{p-1} \nabla$. By the chain rule $D$ becomes $\alpha_{1} D, E$. remains unchanged and a becomes $\alpha_{1}^{p-1}$ a so that $E\left(D^{p-1}-a I\right)$ becomes $\alpha_{1}^{p-1} E\left(D^{p-1}\right.$ $-a I)$.

By $\left(^{*}\right)$ there is a point $Q \in k^{n}$ where the matrix $\left[\begin{array}{ccc}D_{1}\left(f_{1}\right)(Q) & \cdots & D_{n}\left(f_{1}\right)(Q) \\ \vdots & & \vdots \\ D_{1}\left(f_{n-1}\right)(Q) & \cdots & D_{n}\left(f_{n-1}\right)(Q)\end{array}\right]$ is row independent over $k$.

Since $\alpha$ is invariant under a change in coordinates we may assume $Q$ $=(0, \ldots, 0)$. Furthermore since $\alpha$ is clearly unaffected by the constant terms of the $f_{i}$, we may assume that $f_{1}(Q)=\cdots=f_{n}(Q)=0$. We then have that the degree one forms of the $f_{i}$ are $k$-independent. Therefore, after another linear change we may assume that the lowest degree form of $f_{i}$ is $x_{i}(1 \leq i \leq n$ -1). Again apply both sides of $(2.1 .6)$ to $\left(x_{1} \cdots x_{n}\right)^{p-1}$ and compare the 0 degree terms. On the right we get $(-1)^{n} \alpha$ and on the left we get $(-1)^{n}$. Hence $\alpha=1$ in (2.1.6).

Now let $t \in L$. Since $D^{p-1} t-a t \in L^{\prime}$, there are unique $\beta_{\bar{r}} \in L$ such that $D^{p-1} t-a t=\sum_{\bar{r}} \beta_{\bar{r}} f^{\bar{r}} . \quad$ Fix $\bar{s}=\left(s_{1}, \ldots, s_{n-1}\right) \in \mathbb{F}_{p}^{n-1}$. Then on the one hand we know by (2.1.6) with $\alpha=1$ that $E\left(D^{p-1}\left(f^{\bar{s}} t\right)-a f^{\bar{s}} t\right)=\nabla\left(f^{\bar{s}} t\right)$. On the other hand, we have $E\left(D^{p-1}\left(f^{\bar{s}} t\right)-a f^{\bar{s}} t\right)=E\left(f^{\bar{s}}\left(D^{p-1} t-a t\right)\right)=(-1)^{n-1} \beta_{\bar{r}_{0}}$ where $\bar{r}_{0}$ $=\left(p-1-s_{1}, \ldots, p-1-s_{n-1}\right)$, from which (iii) follows,

Case (II). The $f_{i}$ contain no monomials that are $p^{\text {th }}$ powers.

Assume the coefficients of the $f_{i}$ are algebraically independent over $k$. Then $\left(^{*}\right)$ is satisfied and hence the formula in (iii) holds. Therefore it will hold after any specialization of the coefficients, since with respect to the differential operators $D$ and $\nabla$ they are constants. Finally observe that if the $f_{i}$ are replaced by $h_{i}, 1 \leq i \leq n-1$, such that $f_{i}-h_{i} \in B=k\left[x_{1}^{p}, \ldots, x_{n}^{p}\right]$, then $D$ and hence a (such that $D^{p}=a D$ ) remain unchanged. The next lemma shows that the right side of the equality in $(2.1$ iii) also remains unchanged by such a substitution. Case II showed that the desired formula holds whenever the $f_{i}$ contain no $p$-th powers. Thus the general case now follows from the above observations. 
2.2. Lemma. Assume $f_{1}, \ldots, f_{n-1}, h_{1} \in A$ with $f_{1}-h_{1} \in B=k\left[x_{1}^{p}, \ldots, x_{n}^{p}\right]$. Then for all $t \in L$,

$$
\begin{aligned}
\sum_{s_{l}} f_{1}^{s_{1}} \cdots & f_{n-1}^{s_{n-1}} \nabla\left(f_{1}^{p-s_{1}-1} \cdots f_{n-1}^{p-s_{n-1}-1} t\right)= \\
& =\sum_{s_{l}} h_{1}^{s_{1}} f_{2}^{s_{2}} \cdots f_{n-1}^{s_{n-1}} \nabla\left(h_{1}^{p-s_{1}-1} f_{2}^{p-s_{2}-1} \cdots f_{n-1}^{p-s_{n-1}-1} t\right)
\end{aligned}
$$

Proof. $h_{1}=f_{1}+\alpha$, for some $\alpha \in B$. Let $t \in L$ and $t_{0}=f_{2}^{p-s_{2}-1} \ldots$ $f_{n-1}^{p-s_{n-1}-1} t$. Then $\quad \sum_{s_{1}} h_{1}^{s_{1}} f_{2}^{s_{2}} \cdots f_{n-1}^{s_{n-1}} \nabla\left(h_{1}^{p-s_{1}-1} \cdots f_{n-1}^{p-s_{n-1}-1} t\right)=\sum_{\left(s_{2}, \ldots, s_{n-1}\right)} f_{2}^{s_{2}} \cdots$ $f_{n-1}^{s_{n-1}} \sum_{s_{1}=0}^{p-1} h_{1}^{s_{1}} \nabla\left(h_{1}^{p-s_{1}-1} t_{0}\right)$. So it is enough to show that $\sum_{s=0}^{p-1} h^{s} \nabla\left(h^{p-s-1} t\right)$ $=\sum_{s=0}^{p-1} f^{s} \nabla\left(f^{p-s-1} t\right)$, when $h-f=\alpha \in B$.

We have $\sum_{s=0}^{p-1} h^{s} \nabla\left(h^{p-s-1} t\right)$

$$
\begin{aligned}
& =\sum_{s=0}^{p-1} \sum_{i=0}^{s}\left(\begin{array}{l}
s \\
i
\end{array}\right) f^{i} \alpha^{s-i} \sum_{j=0}^{p-1-s}\left(\begin{array}{c}
p-1-s \\
j
\end{array}\right) \alpha^{p-1-s-j} \nabla\left(f^{j} t\right) \\
& =\sum_{s=0}^{p-1} \sum_{i=0}^{s} \sum_{j=0}^{p-1-s}\left(\begin{array}{c}
s \\
i
\end{array}\right)\left(\begin{array}{c}
p-1-s \\
j
\end{array}\right) f^{i} \alpha^{p-1-i-j} \nabla\left(f^{j} t\right) \\
& =\sum_{j=0}^{p-1} \alpha^{-j} \nabla\left(f^{j} t\right) \sum_{s=0}^{p-1}\left(\begin{array}{c}
p-1-s \\
j
\end{array}\right) \alpha^{p-1-s} \sum_{i=0}^{s}\left(\begin{array}{l}
s \\
i
\end{array}\right) f^{i} \alpha^{s-i} \\
& =\sum_{j=0}^{p-1} \alpha^{-j} \nabla\left(f^{j} t\right) \sum_{s=0}^{p-1}\left(\begin{array}{c}
p-1-s \\
j
\end{array}\right) \alpha^{p-1-s}(f+\alpha)^{s} \\
& =\sum_{j=0}^{p-1}(-1)^{j} \nabla\left(f^{j} t\right) \sum_{s=0}^{p-1}\left(\begin{array}{c}
p-1-j \\
s
\end{array}\right)(-1)^{s} \alpha^{p-1-j-s}(f+\alpha)^{s}
\end{aligned}
$$

(Note that $\left(\begin{array}{c}p-1-s \\ j\end{array}\right)\left(\begin{array}{c}p-1 \\ s\end{array}\right)=\left(\begin{array}{c}p-1-j \\ s\end{array}\right)\left(\begin{array}{c}p-1 \\ j\end{array}\right)$

$$
\begin{aligned}
& \text { and } \left.\left(\begin{array}{c}
p-1 \\
j
\end{array}\right)=(-1)^{j}, \text { etc. }\right) \\
= & \sum_{j=0}^{p-1}(-1)^{j}\left(\nabla\left(f^{j} t\right)\right)(\alpha-(f+\alpha))^{p-1-j} \\
= & \sum_{j=0}^{p-1} f^{p-1-j} \nabla\left(f^{j} t\right)=\sum_{s=0}^{p-1} f^{s} \nabla\left(f^{p-1-s} t\right) .
\end{aligned}
$$

The next proposition generalizes ([3], page 74, Theorem (3.4)). In the two 
variable case it was used to prove that a generic Zariski surface has 0-divisor class group ([9]). matrix

Let $S=S\left(f_{1}, \ldots, f_{n-1}\right)=\left\{Q \in k^{n}: \operatorname{rank}\left[D_{i}\left(f_{j}\right)\right](Q)<n-1\right\}$. Let $C$ be the

$$
C=\left[\begin{array}{cccc}
D_{1}\left(f_{1}\right) & \cdots & D_{n-1}\left(f_{1}\right) & D_{n}\left(f_{1}\right) \\
\vdots & & \vdots & \vdots \\
D_{1}\left(f_{n-2}\right) & \cdots & D_{n-1}\left(f_{n-2}\right) & D_{n}\left(f_{n-2}\right) \\
0 & \cdots & 1 & 0 \\
0 & \cdots & 0 & 1
\end{array}\right]
$$

and $C^{*}=\left(C^{-1}\right)^{t}\left(=\right.$ the transpose of $\left.C^{-1}\right)$. Let $\left[g_{1}, \ldots, g_{n}\right]$ and $\left[h_{1}, \ldots, h_{n}\right]$ be the $n-1-s t$ and $n$-th rows of $C^{*}$, respectively. Let $E_{1}^{2}=\sum_{i, i=1}^{n} g_{i} g_{j} \frac{\partial^{2}}{\partial x_{i} \partial x_{j}}, E_{2}^{2}$ $=\sum_{i, j=1}^{n} h_{i} h_{j} \frac{\partial^{2}}{\partial x_{i} \partial x_{j}}$, and $E_{1} E_{2}=\sum_{i, j=1}^{n} g_{i} h_{j} \frac{\partial^{2}}{\partial x_{i} \partial x_{j}}$. Let $M_{i}$ be the cofactor of $D_{n-1}\left(f_{i}\right)$ in the matrix

$$
\left[\begin{array}{ccc}
D_{1}\left(f_{1}\right) & \cdots & D_{n-1}\left(f_{1}\right) \\
\vdots & & \vdots \\
D_{1}\left(f_{n-1}\right) & \cdots & D_{n-1}\left(f_{n-1}\right)
\end{array}\right], 1 \leq i \leq n-1 .
$$

Let $H=\left[\sum_{j=1}^{n-1} M_{j} E_{1} E_{2}\left(f_{j}\right)\right]^{2}-\left[\sum_{j=1}^{n-1} M_{j} E_{1}^{2}\left(f_{j}\right)\right]\left[\sum_{j=1}^{n-1} M_{j} E_{2}^{2}\left(f_{j}\right)\right]$.

2.3. Proposition. For all $Q \in S, a(Q)=(H(Q))^{(p-1) / 2}$, where $a$ is as in (2.1).

Proof. It is a straightforward linear algebra to check that for all $Q \in S$, $g_{i}(Q)$ and $h_{i}(Q)$ are independent of the order of $f_{1}, \ldots, f_{n-1}$ up to a change of the same sign. It follows that $H(Q)$ is independent of the order of $f_{1}, \ldots, f_{n-1}$.

Let $Q \in S$ be a point where the rank $\left[D_{i}\left(f_{j}\right)(Q)\right]=n-2$. After a change of coordinates, which will not alter $D$ (hence $a$ ) or $H$, we may assume $Q$ $=(0, \ldots, 0)$. By the above remark, we may assume $M_{n-1}(Q) \neq 0$. Then

2.3.1 $a_{1} D_{i}\left(f_{1}\right)(Q)+\cdots+a_{n-2} D_{i}\left(f_{n-2}\right)(Q)=D_{i}\left(f_{n-1}\right)(Q), 1 \leq i \leq n$, where

$$
a_{r}=-M_{r}(Q) / M_{n-1}(Q), 1 \leq r \leq n-2 \text {. }
$$

Replacing $f_{j}$ by $f_{j}-f_{j}(Q), 1 \leq j \leq n-1$ also does not change $D$ (and hence $a$ ) or $H$ so that we may assume $f_{j}(Q)=0,1 \leq j \leq n-1$.

Temporarily, we replace $f_{n-1}$ by $f_{n-1}-\sum_{j=1}^{n-2} a_{j} f_{j}$. Then $D$ and a remain unchanged, and after this substitution we have that 


$$
f_{j}(Q)=D_{i}\left(f_{n-1}\right)(Q)=0, \quad 1 \leq j \leq n-1,1 \leq i \leq n .
$$

Now make the change in coordinates

$$
\bar{x}_{i}=\sum_{l=1}^{n} D_{l}\left(f_{i}\right)(Q) \cdot x_{l}, 1 \leq i \leq n-2, \bar{x}_{n-1}=x_{n-1}, \bar{x}_{n}=x_{n} .
$$

Then $Q$ remains $(0, \ldots, 0)$ and $(2.3 .2)$ still holds. By the chain rule we have for all $h \in L$,

$$
D_{i}(h)=\sum_{j=1}^{n-2} D_{i}\left(f_{j}\right)(Q) \cdot h_{\bar{x}_{j}} \quad \text { for } \quad 1 \leq i \leq n-2 .
$$

and

$$
D_{i}(h)=\sum_{j=1}^{n-2} D_{i}\left(f_{j}\right)(Q) \cdot h_{\bar{x}_{j}}+h_{\bar{x}_{i}} \quad \text { for } \quad n-1 \leq i \leq n .
$$

Then $D=$

$$
\begin{aligned}
& \operatorname{det}\left[\begin{array}{ccc}
\sum_{j=1}^{n-2} D_{1}\left(f_{j}\right)(Q) \cdot \frac{\partial}{\partial \bar{x}_{j}} & \cdots \cdots & \sum_{j=1}^{n-2} D_{n-1}\left(f_{j}\right)(Q) \cdot \frac{\partial}{\partial \bar{x}_{j}}+\frac{\partial}{\partial \bar{x}_{n}} \\
\cdots \cdots & \cdots \cdots & \cdots \cdots \\
\sum_{j=1}^{n-2} D_{1}\left(f_{j}\right)(Q) \cdot\left(f_{n-2}\right)_{\bar{x}_{j}} & \cdots \cdots & \sum_{j=1}^{n-2} D_{n-1}\left(f_{j}\right)(Q) \cdot\left(f_{n-2}\right)_{\bar{x}_{j}}+\left(f_{n-2}\right)_{\bar{x}_{n}} \\
\sum_{j=1}^{n-2} D_{1}\left(f_{j}\right)(Q) \cdot\left(f_{n-1}\right)_{\bar{x}_{j}} & \cdots \cdots & \sum_{j=1}^{n-2} D_{n-1}\left(f_{j}\right)(Q) \cdot\left(f_{n-1}\right)_{\bar{x}_{j}}+\left(f_{n-1}\right)_{\bar{x}_{n}}
\end{array}\right] \\
& \operatorname{det}\left[\begin{array}{llll}
\frac{\partial}{\partial \bar{x}_{1}} & \frac{\partial}{\partial \bar{x}_{2}} & \cdots & \frac{\partial}{\partial \bar{x}_{n}} \\
\left(f_{1}\right)_{\bar{x}_{1}} & \left(f_{1}\right)_{\bar{x}_{2}} & \cdots & \left(f_{1}\right)_{\bar{x}_{n}} \\
\cdots & \cdots & \cdots & \cdots \\
\left(f_{n-1}\right)_{\bar{x}_{1}} & \left(f_{n-1}\right)_{\bar{x}_{2}} & \cdots & \left(f_{n-1}\right)_{\bar{x}_{n}}
\end{array}\right]
\end{aligned}
$$

$$
\left[\begin{array}{lllll}
D_{1}\left(f_{1}\right)(Q) & \cdots & D_{n-2}\left(f_{1}\right)(Q) & D_{n-1}\left(f_{1}\right)(Q) & D_{n}\left(f_{1}\right)(Q) \\
D_{1}\left(f_{2}\right)(Q) & \cdots & D_{n-2}\left(f_{2}\right)(Q) & D_{n-1}\left(f_{2}\right)(Q) & D_{n}\left(f_{2}\right)(Q) \\
\vdots & \vdots & \vdots & \vdots \\
D_{1}\left(f_{n-2}\right)(Q) & \cdots & D_{n-2}\left(f_{n-2}\right)(Q) & D_{n-1}\left(f_{n-2}\right)(Q) & D_{n}\left(f_{n-2}\right)(Q) \\
0 & \cdots & 0 & 1 & 0 \\
0 & \cdots & 0 & 0 & 1
\end{array}\right]
$$


$=M_{n-1}(Q)\left[\bar{J}\left(, f_{1}, \ldots, f_{n-1}\right)\right]$, where $\bar{J}$ is the determinant of the jacobian matrix with respect to $\bar{x}, \cdots, \bar{x}_{n}$.

Let $\bar{D}=\bar{J}\left(, f_{1}, \ldots, f_{n-1}\right)$ and $\bar{a}$ be such that $\bar{D}^{p}=\bar{a} \bar{D}$ (see (2.1)).

Then $a=\left(M_{n-1}(Q)\right)^{p-1} \bar{a}$. Then $a(Q)=\left(M_{n-1}(Q)\right)^{p-1} \bar{a}(Q)$. We have by (2.1) and (2.3.2) that $\bar{a}(\bar{Q})=(-1)^{n} \bar{\nabla}\left(\left(f_{1} \cdots f_{n}\right)^{p-1}\right)(Q)$ where $\bar{\nabla}$ $=\frac{\partial^{n(p-1)}}{\partial \bar{x}_{1}^{p-1} \cdots \partial \bar{x}_{n}^{p-1}}$. Also by the change in coordinates (2.3.3) we have

2.3.5. $f_{i}=\bar{x}_{i}+f_{i}^{+}, 1 \leq i \leq n-2$, where $f_{i}^{+}=f_{i}-\left(\right.$ leading form of $f_{i}$ ) and

$$
\begin{aligned}
& f_{n-1}=\frac{1}{2} \sum_{i, j=1}^{n}\left(f_{n-1}\right)_{\bar{x}_{i} \bar{x}_{j}}(Q) \bar{x}_{i} \bar{x}_{j}+f_{n-1}^{+} \text {if } \quad p>2, \\
& \left.f_{n-1}=\sum_{i<j}\left(f_{n-1}\right)_{\bar{x}_{i} \bar{x}_{j}}(Q) \bar{x}_{i} \bar{x}_{j}+f_{n-1}^{+}\right) \text {if } p=2 .
\end{aligned}
$$

Thus the initial form of $\left(f_{1} \cdots f_{n-1}\right)^{p-1}$ is

2.3.6. $\left(\bar{x}_{1} \cdots \bar{x}_{n-2}\right)^{p-1}\left(\left(f_{n-1}\right)_{\bar{x}_{n-1} \bar{x}_{n-1}}(Q) \frac{\bar{x}_{n-1}^{2}}{2}+\left(f_{n-1}\right)_{\bar{x}_{n} \bar{x}_{n}}(Q) \frac{\bar{x}_{n}^{2}}{2}+\left(f_{n-1}\right)_{\bar{x}_{n-1} \bar{x}_{n}}\right.$

$\left.(Q) \cdot \bar{x}_{n-1} \bar{x}_{n}\right)^{p-1}+g$, where $g$ is homogeneous of degree $n(p-1)$, with

$$
\operatorname{deg}_{\bar{x}_{n-1} \bar{x}_{n}}(g)<2(p-1) \text {, if } p>2 \text {. }
$$

If $p=2$, the expression $\left(f_{n-1}\right)_{\bar{x}_{n-1} \bar{x}_{n-1}}(Q) \frac{\bar{x}_{n-1}^{2}}{2}+\left(f_{n-1}\right)_{\bar{x}_{n} \bar{x}_{n}}(Q) \frac{\bar{x}_{n}^{2}}{2}$ is deleted from (2.3.6).

It then follows that if $p>2$,

2.3.7. $\quad \bar{a}(Q)=(-1)^{n} \frac{\partial^{2(p-1)}}{\partial \bar{x}_{n-1}^{p-1} \partial \bar{x}_{n}^{p-1}}$.

$$
\begin{gathered}
{\left[\left(f_{n-1}\right)_{\bar{x}_{n-1} \bar{x}_{n-1}}(Q) \frac{\bar{x}_{n-1}^{2}}{2}+\left(f_{n-1}\right)_{\bar{x}_{n} \bar{x}_{n}}(Q) \frac{\bar{x}_{n}^{2}}{2}+\left(f_{n-1}\right)_{\bar{x}_{n-1} \bar{x}_{n}}(Q) \bar{x}_{n-1} \bar{x}_{n}\right]^{p-1}} \\
=\left[\left(f_{n-1}\right)_{\bar{x}_{n-1} x_{n}}^{2}-\left(f_{n-1}\right)_{\bar{x}^{n-1} \bar{x}_{n-1}}\left(f_{n-1}\right)_{\bar{x}_{n} \bar{x}_{n}}\right]^{(p-1) / 2}(Q) \text { by }
\end{gathered}
$$

(2.4) below. If $p=2, \bar{a}(Q)=\left(f_{n-1}\right)_{\bar{x}_{n-1}-1}^{p \bar{x}_{n}}(Q)$. Therefore $a(Q)=\left[\left(M_{n-1}\left(f_{n-1}\right)\right.\right.$ $\left.\left.\bar{x}_{n-1} \overline{\bar{x}}_{n}\right)^{2}-\left(M_{n-1}\left(f_{n-1}\right)_{\overline{\bar{n}}_{n-1} \bar{x}_{n-1}}\right)\left(M_{n-1}\left(f_{n-1}\right)_{\bar{x}_{n} \bar{x}_{n}}\right)\right]^{(p-1) / 2}(Q)$.

We have that

$$
\left[\begin{array}{l}
\bar{x}_{1} \\
\vdots \\
\bar{x}_{n}
\end{array}\right]=C(Q)\left[\begin{array}{c}
x_{1} \\
\vdots \\
x_{n}
\end{array}\right]
$$


Therefore $\left[\begin{array}{c}\frac{\partial}{\partial \bar{x}_{1}} \\ \vdots \\ \frac{\partial}{\partial \bar{x}_{n}}\end{array}\right]=C^{*}(Q)\left[\begin{array}{c}\frac{\partial}{\partial x_{1}} \\ \vdots \\ \frac{\partial}{\partial x_{n}}\end{array}\right]$

We then obtain

$$
a(Q)=\left[\left(M_{n-1} E_{1} E_{2}\left(f_{n-1}\right)\right)^{2}-\left(M_{n-1} E_{1}^{2}\left(f_{n-1}\right)\right)\left(M_{n-1} E_{2}^{2}\left(f_{n-1}\right)\right)\right]^{(p-1) / 2}(Q)
$$

Now back substitute to replace $f_{n-1}$ by $f_{n-1}+\sum_{r=1}^{n-2} \frac{M_{r}(Q)}{M_{n-1}(Q)} f_{r}$ to obtain $a(Q)=(H(Q))^{(p-1) / 2}$.

Now if $Q$ were a point such that $\operatorname{rank}\left[D_{i}\left(f_{j}\right)(Q)\right]<n-2$ then we could have assumed that the first $n-2$ rows of $\left[D_{i}\left(f_{j}\right)(Q)\right]$ are dependent as well. Then clearly $M_{n-1}(Q)=0$ and by an argument similar to that used above, we obtain $\bar{a}(Q)=0$. Thus we obtain $a(Q)=(H(Q))^{(p-1) / 2}$, for all $Q \in S$.

2.4. Lemma. Let $k$ be a ring of characteristics $p>0$. Let $A, B$ and $C \in k$ and $F=A x^{2}+B y^{2}+C x y \in k[x, y]$. Then $\frac{\partial^{2 p-2}\left(F^{p-1}\right)}{\partial x^{p-1} \partial y^{p-1}}=\left(C^{2}-4 A B\right)^{(p-1) / 2}$.

Proof. The coefficient of $x^{p-1} y^{p-1}$ in $F^{p-1}$ if $p>2$ is

$$
\begin{aligned}
& \sum_{i=0}^{(p-1) / 2}\left(\begin{array}{c}
p-1 \\
2 i
\end{array}\right)\left(\begin{array}{c}
2 i \\
i
\end{array}\right) C^{p-1-2 i}(A B)^{i}=\sum_{i=0}^{(p-1) / 2}(-1)^{2 i}\left(\begin{array}{c}
2 i \\
i
\end{array}\right) C^{p-1-2 i}(A B)^{i} \\
& =\sum_{i=0}^{(p-1) / 2}\left(\begin{array}{c}
2 i \\
i
\end{array}\right) C^{p-1-2 i}(A B)^{i}=\sum_{i=0}^{(p-1) / 2}\left(\begin{array}{c}
2 i \\
i
\end{array}\right)\left(C^{2}\right)^{[(p-1) / 2]-i}(A B)^{i} \\
& =\sum_{i=0}^{(p-1) / 2}(-1)^{i}\left(\begin{array}{c}
(p-1) / 2 \\
i
\end{array}\right) 2^{2 i}\left(C^{2}\right)^{[(p-1) / 2]-i}(A B)^{i} \\
& =\sum_{i=0}^{(p-1) / 2}\left(\begin{array}{c}
(p-1) / 2 \\
i
\end{array}\right)\left(C^{2}\right)^{(p-1) / 2-i}(-4 A B)^{i} \\
& =\left(C^{2}-4 A B\right)^{(p-1) / 2} . \text { If } p=2, \text { the lemma is clearly true. }
\end{aligned}
$$

\section{§3. The Fixed Subring of a Polynomial Ring}

Let $k, A, A^{\prime}, L, L^{\prime}$, and $D=J\left(, f_{1}, \ldots, f_{n-1}\right)$ be as in Section 2. Let $\mathscr{L}$ be the additive group of logarithmic derivatives of $D$ in $A, \mathscr{L}=\left\{h^{-1} D h \in A: h \in\right.$ $L\}$. Assume $\left({ }^{*}\right)$ holds. Let $f=f_{1} \cdots f_{n-1}$. For $I=\left(i_{1}, \cdots, i_{n-1}\right) \in \mathbb{F}_{p}^{n-1}$, let $f^{I}$

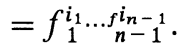

3.1. Lemma. Let $F \subset A_{k}^{2 n-1}$ be the variety defined by the equations $w_{i}^{p}$ 
$=f_{i}\left(x_{1}, \ldots, x_{n}\right), 1 \leq i \leq n-1$. Then the coordinate ring of $F$ is isomorphic to $A^{\prime}$.

Proof. Let $\tilde{A}=k\left[x_{1}, \ldots, x_{n}, w_{1}, \ldots, w_{n-1}\right]$. Let $\phi: \tilde{A} \rightarrow A^{\prime}$ be the ring homomorphism that sends $x_{i}$ to $x_{i}^{p}, w_{j}$ to $f_{j}$ and $\alpha$ to $\alpha^{p}$ for all $1 \leq i \leq n$, $1 \leq j \leq n-1, \quad \alpha \in k$. (Note $\phi$ is not a $k$-homomorphism.) Then $w_{j}^{p}$ $-f_{j} \in \operatorname{ker} \phi$. Let $Q \subseteq \tilde{A}$ be the ideal generated by $w_{j}^{p}-f_{j}, 1 \leq j \leq n$ -1 . Then $Q$ is a prime ideal in $\tilde{A}$ of height $n-1$. Therefore $\operatorname{ker} \phi=Q$ by ([7] page 6,18A).

3.2. Lemma. $D^{-1}(0) \cap A=A^{\prime}$.

Proof. Let $B=D^{-1}(0) \cap A$. Then $k\left[x_{1}^{p}, \ldots, x_{n}^{p}\right] \subset A^{\prime} \subseteq B \subseteq A . \quad B$ is integral over $A^{\prime}$. For each $i, f_{i+1} \notin k\left(x_{1}^{p}, \ldots, x_{n}^{p}, f_{1}, \ldots, f_{i}\right)$ by $\left(^{*}\right)$. Thus $\left[L^{\prime}: k\left(x_{1}^{p}, \ldots, x_{n}^{p}\right)\right]=p^{n-1}$. Also by $\left(^{*}\right), D_{x_{1}} \neq 0$ for some $i$. Therefore the quotient field of $B$ is not $L$ and $A^{\prime}$ and $B$ have the same quotient field. Since $A^{\prime}$ is normal, $A^{\prime}=B$.

3.3. Lemma. $C l\left(A^{\prime}\right) \simeq \mathscr{L}$.

Proof. By $\left({ }^{*}\right)$ the image of $D$ is not contained in any height one prime of $A$. By (1.2) and (3.2), $C l\left(A^{\prime}\right) \simeq \mathscr{L}$ since the units of $A$ are the nonzero elements of $k$.

3.4. Lemma. Let $t \in \mathscr{L}$. Then $\operatorname{deg}(t) \leq M-n$, where $M=\sum_{i=1}^{n-1} \operatorname{deg}\left(f_{i}\right)$.

Proof. $t \in \mathscr{L}$ implies there exists a $g \in L$ such that $g^{-1} D g=t$. Multiplying $g$ by an element of $A^{p}$, if necessary, we may assume $g \in A$. $\operatorname{deg}(D g) \leq(\operatorname{deg} g$ $-1)+\sum_{i=1}^{n-1} \operatorname{deg}\left(f_{i}-1\right)=\operatorname{deg} g+M-n . \quad$ Therefore $\operatorname{deg}(t) \leq M-n$.

3.5. Lemma. Let $\mathscr{G}=\left\{t \in A: \nabla\left(f^{p-1} t\right)=(-1)^{n} t^{p}\right\}$, where $f=f_{1} \cdots f_{n-1}$ and $\nabla$ $=D_{1}^{p-1} \cdots D_{n}^{p-1}$. Then $\mathscr{G}$ is a p-group of type $(p, \ldots, p)$ of order $p^{N}$ with $N \leq\left(\begin{array}{c}M \\ n\end{array}\right)$

Proof. Let $t \in \mathscr{G} . \quad \nabla\left(f^{p-1} t\right)=(-1)^{n} t^{p} \quad$ implies $\quad p \operatorname{deg} t \leq(p-1) \operatorname{deg}(f)$ $+\operatorname{deg}(t)-n(p-1)$. Thus $\operatorname{deg}(t) \leq M-n$. Write $t=\sum_{|J| \leq M-n} \alpha_{J} x^{J}$ where for $J$ $=\left(i_{1}, \ldots, i_{n}\right) \in\left(\mathbb{Z}^{+}\right)^{n}, x^{J}=x_{1}^{i_{1}} \cdots x_{n}^{i_{n}}$ and $|J|=\sum_{j=1}^{n} i_{j}$. Comparing coefficients on both sides of the quality $\nabla\left(f^{p-1} t\right)=(-1)^{n} t^{p}$ we obtain for each $J_{0}$ with $\left|J_{0}\right| \leq M-n$ an equation of the form $L_{J_{0}}=\alpha_{J_{0}}^{p}$, where $L_{J_{0}}$ is a linear expression in the $\alpha_{J}$ with coefficients in $k$.

There are a total of $\left(\begin{array}{c}n+(M-n) \\ n\end{array}\right)=\left(\begin{array}{c}M \\ n\end{array}\right)$ such equations. The ring $R$ 
$=k\left[\alpha_{J}\right]_{|J| \leq M-n}$ with the relations $L_{J}=\alpha_{J}^{p}$ is a finite dimensional $k$-vector spaced spanned by all monomials in the $\alpha_{J}$ of degree less than or equal to $(p-1)$ $\left(\begin{array}{c}M \\ n\end{array}\right)$. Thus $R$ has a finite number of maximal ideals ([10], p. 89).

Thus the $\left(\begin{array}{c}M \\ n\end{array}\right)$ equations $L_{J}=\alpha_{J}^{p}$ intersect at a finite number of points. There is no solution to these equations at infinity. By Bezout's Theorem this number is at most $p^{\left({ }_{n}^{M}\right)}([6]$, p. 670). "Therefore $\mathscr{G}$ is of order at most $p^{\left(\begin{array}{c}M \\ n\end{array}\right)} . \quad \mathscr{G}$ is a $p$-group of type $(p, \ldots, p)$ since $\mathscr{G} \subset A$.

3.6. Proposition. Let $F \subset A_{k}^{2 n-1}$ be the variety defined by $w_{i}^{p}=f_{i}\left(x_{1}, \ldots, x_{n}\right)$, $1 \leq i \leq n-1$. Then $C l(F)$ is a finite $p$-group of type $(p, \ldots, p)$ of order $p^{N}$ where $N \leq\left(\begin{array}{c}M \\ n\end{array}\right)$.

Proof. By (1.2), (3.1), (3.2), and (3.3), $C l(F) \simeq \mathscr{L}$. By (1.3b) an element $t \in A$ is in $\mathscr{L}$ if and only if $D^{p-1} t-a t=-t^{p}$. By (2.1) $t \in \mathscr{L}$ if and only if 3.6.1.

$$
\begin{aligned}
& \nabla\left(f^{p-1} t\right)=(-1)^{n} t^{p} \text { and } \nabla\left(f^{J} t\right)=0 \\
& \qquad \text { for all } J \in \mathbb{F}_{p}^{n-1} \text { with } J \neq(p-1, \ldots, p-1) .
\end{aligned}
$$

Thus $\mathscr{L} \subset \mathscr{G}$. Now use (3.5).

3.7. Lemma. Let $f \in A$ be such that $s^{-1} D s \in A$. Assume $s=g^{r} h$, where $g \in A$ is irreducible, $r \neq 0(\bmod p)$ is a positive integer and $h \in A$ is relatively prime to g. Then $g^{-1} D g \in A$.

Proof. Let $t=s^{-1} D s$. Then $s t=D s=r g^{r-1} h D g+g^{r} D(h)$. Then $g$ divides $r h D g$ and hence $g$ divides $D g$,

\section{§4. Examples}

4.1. Remark. From the proof of (3.6) we see that the calculation of $C l(X)$ is equivalent to determining the number of solutions to a corresponding system of equations of the form

4.1.1. $L_{J}=\alpha_{J}^{p}, L_{J^{\prime}}=0$ where the $J, J^{\prime} \in \mathbb{F}_{p}^{n-1}$ and the $L_{J}$ and $L_{J^{\prime}}$ are linear expressions in the $\alpha_{J}$.

[1] provides an algorithm for finding the number of solutions to such a $p$ linear system of equations and a computer program for determining this number when the coefficients of the $f_{i}$ belong to a finite field, so that the computation of $\mathrm{Cl}(\mathrm{F})$ in this case is a programmable process.

4.2. Remark. Let $h_{i}, 1 \leq i \leq n-1$, be homogeneous elements of $A$ of degree $s_{i}$ 
with $s_{i} \neq 0(\bmod p)$. If the $h_{i}$ satisfy $\left({ }^{*}\right)$, then for each pair $(i, j)$ with $i \neq j, h_{i}$ and $h_{j}$ have no common factors in $A$ and each $h_{i}$ has no multiple factors in $A$. Let $X \subset A_{k}^{2 n-1}$ be defined by the equations $w_{i}^{p}=h_{i}\left(x_{1}, \ldots, x_{n}\right) 1 \leq i \leq n-1$. The next example studies $C l(X)$.

4.3. Example. By (4.2) each $h_{i}=H_{i 1} \cdots H_{i r_{i}}$, where the $H_{i j}$ are distinct irreducible homogeneous elements of $A$. Let $D=J\left(, h_{1}, \cdots, h_{n-1}\right)$ and $\mathscr{L}$ the group of logarithmic derivatives of $D$ in $A$. Let $h=h_{1} \cdots h_{n-1}$ and $M$ $=\operatorname{deg} h . \quad$ Let $t \in \mathscr{L}$. By (3.6.1)

4.3.1.

$$
\nabla\left(h^{p-1} t\right)=(-1)^{n} t^{p} .
$$

Assume that the lowest degree form of $t$ is of degree $s$ and the highest degree form of $t$ is of degree $m$. Compare the lowest and highest degree forms on both sides of the equality in (4.3.1) we obtain $p s \geq(p-1) M+s-n(p-1)$ and $p m \leq(p-1) M+m-n(p-1)$. Then $m \leq M-n \leq s$ and hence $t$ is homogeneous of degree $M-n$. Repeat the same argument used in the proof of (3.5) to obtain $|C l(X)|=p^{s}$ with $s \leq\left(\begin{array}{c}M-1 \\ n-1\end{array}\right)$.

Now assume that the $h_{i}$ satisfy the additional condition that the variety $Y \subseteq A_{k}^{n} \quad$ defined by $h_{1}=\cdots=h_{n-1}=0$ has a finite number of singularities. (When $n=2$, this condition is implied by $\left({ }^{*}\right)$.) For each pair $(i, j)$, $1 \leq i \leq n-1,1 \leq j \leq r_{i}$, let $t_{i j}=H_{i j}^{-1} D\left(H_{i j}\right)$. By (3.7), $t_{i j} \in \mathscr{L}$ for each $(i, j)$.

4.3.2. Claim. The $t_{i j}$ are $\mathbb{F}_{p}$-independent.

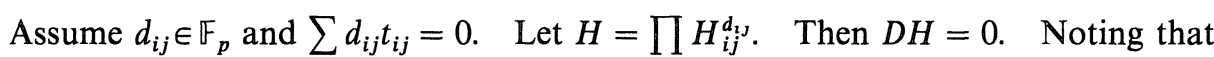
$D h_{i}=0(1 \leq i \leq n-1)$, we may assume that $d_{1} r_{1}=\ldots=d_{n-1} r_{n-1}=0$. By Euler's formula the determinant of the matrix

$$
\left.\begin{array}{ccccccc}
D_{1}(H) & \cdots & D_{j-1}(H) & s H & D_{j+1}(H) & \cdots & D_{n}(H) \\
D_{1}\left(h_{1}\right) & \cdots & D_{j-1}\left(h_{1}\right) & s_{1} h_{1} & D_{j+1}\left(h_{1}\right) & \cdots & D_{n}\left(h_{1}\right) \\
\vdots & & \vdots & \vdots & \vdots & & \vdots \\
D_{1}\left(h_{n-1}\right) & \cdots & D_{j-1}\left(h_{n-1}\right) & s_{n-1} h_{n-1} & D_{j+1}\left(h_{n-1}\right) & \cdots & D_{n}\left(h_{n-1}\right)
\end{array}\right]
$$

is 0 for each $j=1, \ldots, n$, where $s=\operatorname{deg}(H)$.

This shows that either $s \equiv 0(\bmod p)$ or $Y$ has an infinite number of singularities satisfying the equation $H_{1 r_{1}}=\cdots=H_{n-1 r_{n-1}}=0$. Thus $s \equiv 0$ $(\bmod p)$.

If some $d_{i j} \neq 0$ we may assume without loss of generality that $d_{11} \neq 0$. Let $H_{0}=h_{1}^{p-d_{11}} H$. Let $H^{\prime}$ be obtained from $H_{0}$ by factoring out all $p$-th powers. Then $\operatorname{deg}\left(H^{\prime}\right) \equiv s_{1}\left(p-d_{11}\right) \not \equiv 0 \quad(\bmod p)$ and the factors $H_{11}$, 
$H_{2 r_{2}}, \ldots, H_{n-1 r_{n-1}}$ do not appear in $H^{\prime}$. Repeat the above argument to obtain $d_{i j}=0$ for all $2 \leq i \leq n-1$ and that the exponent of $H_{1 r_{1}}$ in $H^{\prime}$ must also be 0 . But this implies that $p-d_{11}=0$. Contradiction!

Thus if we let $m$ be the number of factors in $h=h_{1} \cdots h_{n-1}$ we have that the order of $C l(X)$ is $p^{r}$ for some $r$ with $m-n+1 \leq r \leq\left(\begin{array}{c}M-1 \\ n-1\end{array}\right)$.

4.4. Remark. When $n=2,(4.3)$ implies that $r=m-1$, which was first proved in [8].

4.5. Example. Let $f_{i}\left(x_{n}\right), g_{i}\left(x_{n}\right) \in k\left[x_{n}\right], 1 \leq i \leq n-1$. Let $f\left(x_{n}\right)=f_{1}\left(x_{n}\right) \cdots f_{n-1}$ $\left(x_{n}\right)$. Assume $f\left(x_{n}\right)$ has $r$ distinct roots, $\theta_{1}, \ldots, \theta_{r}$. For each $i$, let $F_{i}=x_{i} f_{i}\left(x_{n}\right)$ $+x_{n} g_{i}\left(x_{n}\right)$. Assume the $F_{i}$ satisfy $\left(^{*}\right)$. Let $D=J\left(, F_{1}, \ldots, F_{n-1}\right)$ and $\mathscr{L}$ be the group of logarithmic derivatives of $D$ in $A$. We will show that $\mathscr{L}$ is of order $p^{r}$ generated by the logarithmic derivatives, $D\left(x_{n}-\theta_{j}\right) /\left(x_{n}-\theta_{j}\right), 1 \leq j \leq r$ in $A$. Thus the group of Weil divisors of the variety defined by the equations, $w_{i}^{p}$ $=F_{i}, 1 \leq i \leq n-1$, will be a direct sum of $r$ copies of $\mathbb{F}_{p}$.

Let $t \in \mathscr{L}$. Given $h \in A, \operatorname{deg}_{x_{1}}(D h) \leq \operatorname{deg}_{x_{t}}(h), 1 \leq i \leq n-1$. Therefore $\operatorname{deg}_{x_{i}}(t)=0$ for $1 \leq i \leq n-1$. Thus $t \in k\left[x_{n}\right]$. Let $\Delta$ be the $k$-derivation on $k\left(x_{1}, \ldots, x_{n}\right)$ defined by $\Delta=t^{-1} D$. By Hochschild's formula ([11], pg. 64, (3.2)), $\Delta^{p}=\Delta$. Hence $(\Delta-(p-1) I) \cdots(\Delta-2 I)(\Delta-I) \Delta=\Delta^{p}-\Delta=0$, where $I$ is the identity mapping of $k\left(x_{1}, \ldots, x_{n}\right)$ into $k\left(x_{1}, \ldots, x_{n}\right)$. Clearly $\Delta\left(x_{n}\right) \neq 0$. Set $y_{1}$ $=\Delta\left(x_{n}\right), \quad y_{2}=(\Delta-I) y_{1}, \ldots, y_{p}=(\Delta-(p-1) I) y_{p-1}(=0)$. First we observe that if $x \in k\left(x_{n}\right)$ then $\Delta(x) \in k\left(x_{n}\right)$. Hence $y_{1}, \ldots, y_{p-1} \in k\left(x_{n}\right)$. Next we have that for some $l=2, \ldots, p-1, y_{l-1} \neq 0$ and $y_{l}=(\Delta-(l-1) I) y_{l-1}=0$. Therefore $\Delta\left(y_{l-1}\right)=(l-1) y_{l-1}$, which implies that $D\left(y_{l-1}\right) / y_{l-1}=(l-1) t$. Let $q$ be the inverse of $l-1$ modulo $p$. Let $y=y_{l-1}^{q}$. Then $D(y) / y=t$. Thus we've shown that there exists $y \in k\left(x_{n}\right)$ such that $D y / y=t$. Multiplying $y$ by an element of $k\left[x_{n}^{p}\right]$, if necessary, we may assume $y \in k\left[x_{n}\right]$.

Factor $y$ into a product of linear factors, $y=\left(x_{n}-\alpha_{1}\right)^{s_{1}} \cdots\left(x_{n}-\alpha_{m}\right)^{s_{m}}$ where $\alpha_{1}, \ldots, \alpha_{m} \in k$ are pairwise distinct. If $s_{i} \geq p$ for some $s_{i}$, then $\left(x_{n}-\alpha_{i}\right)^{-p_{y}}$ will yield the same logarithmic derivative as $\mathrm{y}$, so we may assume that $1 \leq s_{i} \leq p-1$ for each $s_{i}$. By (3.7), $D\left(x_{n}-\alpha_{i}\right) / x_{n}-\alpha_{i} \in \mathscr{L}$ for each $i=1, \ldots, m$. But for each $i, D\left(x_{n}-\alpha_{i}\right)=D\left(x_{n}\right)=(-1)^{n+1} f\left(x_{n}\right)$. Therefore $x_{n}-\alpha_{i}$ is a factor of $f\left(x_{n}\right)$ in $k\left[x_{n}\right]$. We conclude that $\alpha_{i} \in\left\{\theta_{1}, \ldots, \theta_{r}\right\}$ for each $i=1, \ldots, m$. Thus $t$ $=D(y) / y=\sum_{i=1}^{m} s_{i}\left(D\left(x_{n}-\alpha_{i}\right) /\left(x_{n}-\alpha_{i}\right)\right)$ belongs to the $\mathbb{F}_{p}$-space spanned by $\{D$ $\left.\left(x_{n}-\theta_{i}\right) /\left(x_{n}-\theta_{i}\right): 1 \leq i \leq r\right\}$. These polynomials are easily seen to be $\mathbb{F}_{p^{-}}$ independent. Thus $\mathscr{L}$ has order $p^{r}$. 


\section{§5. Purely Inseparable Covers of Dimension Two Factorial Domains}

Let $g, f_{1}, \ldots, f_{n-2} \in A=k\left[x_{1}, \ldots, x_{n}\right]$, where $k$ is algebraically closed of characteristic $p \neq 0$. Let $D=J\left(, g, f_{1}, \ldots, f_{n-2}\right)$. Assume that the ideal $P$ $=\left(f_{1}, \ldots, f_{n-2}\right)$ is a height $n-2$ prime ideal in $A$. Let $B=A / P$. For $f \in A$, denote its image in $B$ by $\bar{f}$. Then $B=k\left[\bar{x}_{1}, \ldots, \bar{x}_{n}\right]$. Let $C=B^{p}[\bar{g}]$ $=k\left[\bar{x}_{1}^{p}, \ldots, \bar{x}_{n}^{p}, \bar{g}\right]$. Denote by $\bar{L}$ and $\bar{K}$ the quotient field of $B$ and $C$, respectively. $D$ will induce a $k$-derivation, $\bar{D}$, on $\bar{L}$. Throughout this section assume (**) (See (0.10).). Let $W \subseteq A_{k}^{n+1}$ be the variety defined by the equations $f_{1}=\cdots=f_{n-2}=w^{p}-g=0$.

5.1. Lemma. (i) $\bar{D}^{-1}(0) \cap B=C$, (ii) $C$ is isomorphic to the coordinate ring of $W$, (iii) $[\bar{L}: \bar{K}]=p$, (iv) $\bar{D}(B)$ is not contained in any height one prime of $B$.

Proof. Consider the surjection $\phi: A[w] \rightarrow C$ given by $x_{i} \rightarrow \bar{x}_{i}^{p}, 1 \leq i \leq n, w$ $\rightarrow \bar{g}$, and $\alpha \rightarrow \alpha^{p}$, for all $\alpha \in k$. Then the ideal $I \subseteq A[w]$ generated by $f_{1}, \ldots, f_{n-2}, w^{p}-g$ is contained in ker $\phi$ and is a prime ideal of height $n-1$ since $\bar{g} \neq \bar{h}^{p}$ for any $\bar{h} \in B$ by assumption. Since the dimension of $C$ is 2 , the height of $\operatorname{ker} \phi$ is $n-1$. Thus $A[w] / I \cong C$, which proves (ii).

We have $B^{p} \subseteq C \subseteq \bar{D}^{-1}(0) \cap B \subseteq B$ and $\left[\bar{K}: \bar{L}^{p}\right]=p$. By lemma (5.2)(below), $\left[\bar{L}: \bar{L}^{p}\right]=p^{2}$. Therefore $C$ and $\bar{D}^{-1}(0) \cap B$ have the same quotient field. By (ii) $C$ is normal, which gives $C=\bar{D}^{-1}(0) \cap B$. Also $[\bar{L}: \bar{K}]=\left[\bar{L}: \bar{L}^{p}\right]$ $/\left[\bar{K}: \bar{L}^{p}\right]=p$. Hence (iii).

(iv) is immediate from the assumption on $W$.

5.2. Lemma. Let $k$ be a perfect field of characteristic $p \neq 0$. Let $A$ be $a$ finitely generated $k$-integral domain of dimension 2. Let $B=A^{p}$. Then the degree of $A$ over $B$ is $p^{2}$.

Proof. $A=k\left[u_{1}, \ldots, u_{n}\right]$ for some $u_{i} \in A$. Then $B=k\left[u_{1}^{p}, \ldots, u_{n}^{p}\right]$. By Noether's normalization theorem there exists $y_{1}, y_{2} \in A$ such that $A$ is separably algebraic over $k\left[y_{1}, y_{2}\right]$ and $y_{1}, y_{2}$ are algebraically independent over $k$. We then have the diagram of inclusions

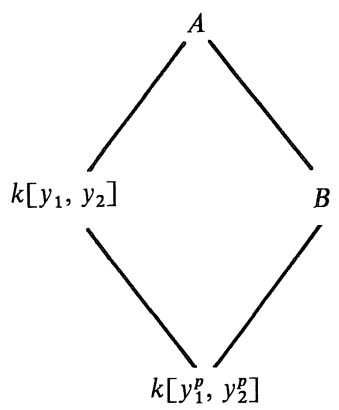


Let $L, L^{\prime}$ be the quotient fields of $A, B$, respectively. Clearly [L: $\left.k\left(y_{1}, y_{2}\right)\right]$ $=\left[L^{\prime}: k\left(y_{1}^{p}, y_{2}^{p}\right)\right]$ and the result follows.

5.3. Corollary. Let $C$ be as in (5.1). $C l(C) \cong \overline{\mathscr{L}} / \overline{\mathscr{L}}^{\prime}$, where $\overline{\mathscr{L}}$ $=\left\{\bar{f}^{-1} \bar{D}(\bar{f}) \in B\right\}, \overline{\mathscr{L}}^{\prime}=\left\{\bar{u}^{-1} D(\bar{u}): \bar{u}\right.$ is a unit in $\left.B\right\}$.

Proof. Use (5.1) and (1.2).

Throughout the remainder of this section assume that each $f_{i}$ is homogeneous of degree $s_{i}, 1 \leq i \leq n-2$ and $g$ is homogeneous of degree $s \neq 0$ $(\bmod p) . \quad$ Let $M=s+\sum_{i=1}^{n-2} s_{i}$.

5.4. Lemma. Let $\bar{w} \in \overline{\mathscr{L}}$. Then there exists homogeneous $t \in A$ of degree $M-n$ such that $\bar{t}=\bar{w}$.

Proof. Let $w \in A$ be a representative of $\bar{w}$ of minimal degree. Let $\operatorname{deg}(w)$ $=d$. Then $w=\sum_{j=0}^{d} w_{j}$, where $w_{j} \in A$ is homogeneous of degree $j$. Note $\bar{w}_{d} \neq 0$ by minimality. Let $a \in A$ be such that $D^{p}=a D$. Then $\bar{D}^{p}=\bar{a} \bar{D}$. By (1.3) $\bar{D}^{p-1}(\bar{w})-\bar{a} \bar{w}+\bar{w}^{p}=0 . \quad$ Then $\sum_{j=0}^{d}\left(D^{p-1}\left(w_{j}\right)-a w_{j}+w_{j}^{p}\right) \in P . \quad P$ being homogeneous implies that $w_{d} \in P$ or $D^{p-1}\left(w_{j}\right)-a w_{j}+w_{d}^{p} \in P$ for some $j=0,1, \ldots, d$ with $\operatorname{deg}\left(D^{p-1} w_{j}-a w_{j}\right)=\operatorname{deg}\left(w_{d}^{p}\right)$. (Note if $h$ is homogeneous of degree $r$, then $D^{p-1} h-a h$ is homogeneous of degree $(p-1)(M-n)+r$ or $D^{p-1} h-a h$ $=0$.) Since $\bar{w}_{d} \neq 0$, it must be that $p d=(p-1)(M-n)+j$ for some $j$ $=0,1, \ldots, d$. Then $p d \leq(p-1)(M-n)+d$, which implies that $d \leq M-n$.

5.4.1. The two sets, $\left\{j: \bar{D}^{p-1} \bar{w}_{j}-\bar{a} \bar{w}_{j} \neq 0\right\}$ and $\left\{j: \bar{w}_{j} \neq 0\right\}$, have the same number of elements since $\sum_{j=0}^{d} \bar{D}^{p-1} \bar{w}_{j}-\bar{a} \bar{w}_{j}+\bar{w}_{j}^{p}=0$ and $P$ is homogeneous.

This shows that $\bar{D}^{p-1} \bar{w}_{d}-\bar{a} \bar{w}_{d} \neq 0$. (Note $\bar{w}_{j=0} \Rightarrow \bar{D}^{p-1} \bar{w}_{j}-\bar{a} \bar{w}_{j}=0$.) Therefore $D^{p-1} w_{d}-a w_{d}$ and $w_{d}^{p}$ have the same degree and $\bar{D}^{p-1} \bar{w}_{d}-\bar{a} \bar{w}_{d}+\bar{w}_{d}^{p}$ $=0$. Thus $\bar{w}_{d} \in \overline{\mathscr{L}}$ by (1.3). Then $\bar{w}-\bar{w}_{d}=\sum_{j=1}^{d-1} \bar{w}_{j} \in \overline{\mathscr{L}}$. Repeat the same argument beginning with (5.4.1) to obtain $\bar{w}_{j} \in \overline{\mathscr{L}}, j=1, \ldots, d$. If $\bar{w}_{j} \neq 0$ then this implies that $D^{p-1} w_{j}-a w_{j}$ and $w_{j}^{p}$ have the same degree, but this is only possible if $j=M-n$. Thus it must be that $d=M-n, \bar{w}_{j}=0$ for $j<M-n$ and $\bar{w}=\bar{w}_{d}$.

5.5. Lemma. Let $\bar{w} \in \overline{\mathscr{L}}$. Then there exists homogeneous $y \in A$ such that $\bar{y} \neq 0$ and $\bar{y}^{-1} \bar{D}(\bar{y})=\bar{w}$.

Proof. By (5.4) we may assume $w$ is homogeneous of degree $M-n$. $\bar{\Delta}$ $=\bar{w}^{-1} \bar{D}$. By $\left({ }^{* *}\right), \quad \bar{D}\left(\bar{x}_{r}\right) \neq 0$ for some $r=1, \ldots, n$. Let $\bar{y}_{1}=\bar{\Delta}\left(\bar{x}_{r}\right)$. For 
$2 \leq j \leq p-1$, let $\bar{y}_{j}=\bar{\Delta}\left(\bar{y}_{j-1}\right)-(j-1) \bar{y}_{j-1}$. Then for some $j, \bar{y}_{j}^{-1} \bar{D}\left(\bar{y}_{j}\right)=(j$ $-1) \bar{w}$ by $([11], \operatorname{pg} 64)$, proof of (3.2)). Note also that $\bar{y}_{j}$ is of the form $\bar{v}^{-1} \bar{u}$ where $u, v \in A$ are homogeneous with $\operatorname{deg}(u)=\operatorname{deg}(v)+1$. Multiply $\bar{y}_{j}$ by $\bar{v}^{p}$ to obtain a homogeneous $h \in A$ such that $\bar{h}^{-1} \bar{D}(\bar{h})=(j-1) \bar{w}$. Choose $m \in \mathbb{F}_{p}$ such that $m(j-1)=1$. Then $y=h^{m}$ has the desired property.

5.6. Remark. Assume that $B$ is a unique factorization domain and that $\bar{y} \in B$ is irreducible homogeneous such that $\bar{w}=\bar{y}^{-1} \bar{D}(\bar{y}) \in \overline{\mathscr{L}}$ and $\bar{w} \neq 0$. Then $\bar{x}_{1} \bar{w} \bar{y}=$

$$
\begin{aligned}
\operatorname{det}\left[\begin{array}{cccc}
\bar{x}_{1} \bar{D}_{1}(\bar{y}) & \bar{D}_{2}(\bar{y}) & \cdots & \bar{D}_{n}(\bar{y}) \\
\bar{x}_{1} \bar{D}_{1}(\bar{g}) & \bar{D}_{2}(\bar{g}) & \cdots & \bar{D}_{n}(\bar{g}) \\
\vdots & \vdots & & \vdots \\
\bar{x}_{1} \bar{D}_{1}\left(\bar{f}_{n-2}\right) & \bar{D}_{2}\left(\bar{f}_{n-2}\right) & \cdots & \bar{D}_{n}\left(\bar{f}_{n-2}\right)
\end{array}\right] \\
=\operatorname{det}\left[\begin{array}{cccc}
e \bar{y} & \bar{D}_{2}(\bar{y}) & \cdots & \bar{D}_{n}(\bar{y}) \\
s \bar{g} & \bar{D}_{2}(\bar{g}) & \cdots & \bar{D}_{n}(\bar{g}) \\
\vdots & & & \vdots \\
s_{n-2} \bar{f}_{n-2} & \bar{D}_{2}\left(\bar{f}_{n-2}\right) & \cdots & \bar{D}_{n}\left(\bar{f}_{n-2}\right)
\end{array}\right]
\end{aligned}
$$

by Euler's formula, where $e=\operatorname{deg}(y)$.

Therefore $\bar{x}_{1} \bar{w} \bar{y}=e \bar{y} M_{11}+s \bar{g} M_{21}$, where $M_{11}$ and $M_{21}$ are the cofactors of $e \bar{y}$ and $s \bar{g}$ in the matrix. Thus $\bar{y}$ divides $\bar{g}$ or $M_{21}$ (recall $s \neq 0$ ). Similarly, if $\bar{y}$ does not divide $\bar{g}$, then $\bar{y}$ divides $M_{2 j}, 1 \leq j \leq n$.

Let $\Delta_{j}(1 \leq j \leq n)$ be the derivation on $B$ defined by $\Delta_{j}=$

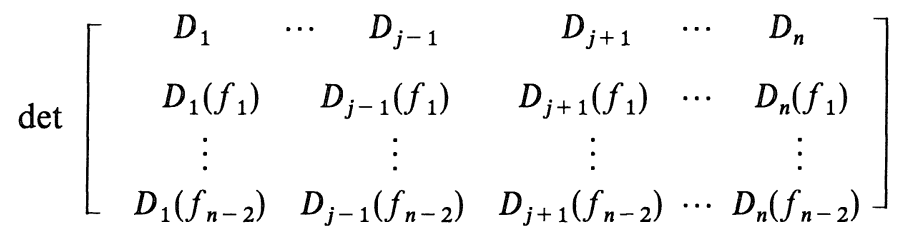

Then $\bigcap_{j=1}^{n} \Delta_{j}^{-1}(0) \cap B=B^{p} \quad$ since $\quad \bar{g} \notin \bigcap_{j=1}^{n} \Delta_{j}^{-1}(0) \cap B \quad$ by $\quad$ (5.1). Also $\bar{y}^{-1} \Delta_{j}(\bar{y}) \in B$ for $1 \leq j \leq n$. At this point, in order to arrive at a definitive description of $C l(W)$ analogous to ([1], page 398, (3.2)), a condition must be added to $\left(^{* *}\right)$ to exclude the possibility that $\bar{y}^{-1} \Delta_{j}(\bar{y}) \notin B(1 \leq j \leq n)$. Hence

5.7. Theorem. Suppose B is a unique factorization domain and that $\bar{g}$ factors in $B$ into a product of $q+1$ distinct prime elements. Assume that either

(i) for each $i=1, \ldots, n, \bar{x}_{i} \notin B^{p}$ and the variety defined by the equations $w^{p}-x_{i}$ $=f_{1}=\cdots=f_{n-2}=0$ in $A_{k}^{n+1}$ defines a unique factorization domain, or

(ii) $\operatorname{End}_{C}(B)=B[G]$, where $B[G]$ denotes the $C$-subalgebra of $\operatorname{End}_{C}[B]$ generated by $B$ and $G=\left\langle\Delta_{1}, \ldots, \Delta_{n}\right\rangle$. 
Then the divisor class group of $W$ is a direct sum of $q$ copies of $\mathbb{Z} / p \mathbb{Z}$.

Some preliminary lemmas are required.

5.8. Lemma. Assume that $B$ is a unique factorization domain and that $\bar{g}$ factors in $B$ into a product of $q+1$ distinct prime elements. Then there exists homogeneous polynomials $g_{1}, \ldots, g_{q+1} \in A$ such that the decomposition of $\bar{g}$ in $B$ into prime elements is given by $\bar{g}=\bar{g}_{1} \cdots \bar{g}_{q+1}$.

Proof. Suppose $\bar{g}=\bar{w}_{1} \bar{w}_{2}$ for some $\bar{w}_{1}, \bar{w}_{2} \in B$. We'll show that we may choose the representatives $w_{1}, w_{2}$ so that they are homogeneous in $A$. Let $w_{1}$ $=u_{0}+\cdots+u_{d}, w_{2}=v_{0}+\cdots+v_{d}^{\prime}$, where $u_{i}, v_{i}$ denote the forms of $w_{1}, w_{2}$ of degree $i$ and $j$, respectively. Then $g-w_{1} w_{2} \in P$. Let $r=\operatorname{deg}(g)$. Then $\sum_{i+j=e} u_{i} v_{j} \in P$ for all $0 \leq e \leq d+d^{\prime}$ with $e \neq r$. Let $i_{0}$ be minimal such that $u_{i_{0}} \notin P$ and $j_{0}$ be minimal such that $v_{j_{0}} \notin P$. Let $i_{0}+j_{0}=m$. Then $\sum_{i+j=m} \bar{u}_{i} \bar{v}_{j}$ $=\bar{u}_{i_{0}} \bar{v}_{j_{0}} \neq 0$, which shows that $m=r$ and $\bar{g}=\bar{u}_{i_{0}} \bar{v}_{j_{0}}$.

5.9. Lemma。 $\mathscr{L}^{\prime}=0$.

Proof. Let $\bar{w} \in \overline{\mathscr{L}}^{\prime}$. By (5.5), there exists a homogeneous element $h \in A$ such that $\bar{h} \neq 0$ and $\bar{h}^{-1} \bar{D}(\bar{h})=\bar{w}$ in $B$. Also by definition of $\overline{\mathscr{L}}^{\prime}$ there is a unit $\bar{u}$ in $B$ such that $\bar{u}^{-1} \bar{D}(\bar{u})=\bar{w}$. Let $\bar{v}=\bar{u}^{-1}$. Then $\bar{D}(\bar{v} \bar{h})=0$. Thus by (5.1), $\bar{v} \bar{h}=\sum_{j=0}^{p-1} \bar{\alpha}_{j}^{p} \bar{g}^{j}\left(\alpha_{j} \in A\right)$. Let $v \in A$ be a preimage of $\bar{v}$. Then $v h-\sum_{j=0}^{p-1} \alpha_{j}^{p} g^{j} \in P$.

Write $v=\sum_{i=0}^{r} v_{i}$ with $v_{i}$ the form of $v$ of degree $i$. $\bar{v}$ being a unit implies $v_{0} \neq 0$. Since $h$ and $g$ are homogeneous and $P$ is a homogeneous ideal and $\operatorname{deg}(g) \neq 0(\bmod p)$, we see by comparing lowest degree forms of $v h$ and $\sum_{j=0}^{p-1} \alpha_{j}^{p} g^{j}$ that for some $\beta \in A$ and $j=, \ldots, p-1, \quad v_{0} h-\beta^{p} g^{j} \in P$. Therefore $\bar{v}_{0} \bar{h} \in C$. Since $v_{0}\left(\right.$ hence $\left.\bar{v}_{0}\right) \in k, \bar{h} \in C$ and $\bar{w}=\bar{h}^{-1} D(\bar{h})=0$ by $(5.1)$.

Proof of theorem (5.7): Continuing with (5.6), we have $\bar{y}^{-1} \Delta_{j}(\bar{y}) \in B$ for $1 \leq j \leq n$. Since $\bar{D}(\bar{y}) \neq 0, \Delta_{j}(\bar{y}) \neq 0$ for some $j$. If we assume (i), then either the divisor class group of the variety defined by the equations $w^{p}-x_{j}=f_{1}=\cdots$ $=f_{n-2}$ is not trivial or $\bar{y}$ is a unit in $B$ by (1.2), which contradicts the irreducibility of $\bar{y}$ in $B$. If we assume (ii) then either $C l\left(B^{p}\right) \neq 0$ or $\bar{y}$ is a unit in $B$ by theorem $([4]$, page $93,(17.4)$ ). Thus in either case, $\bar{y}$ is a factor of $\bar{g}$.

Let $\bar{g}=\bar{g}_{1} \cdots \bar{g}_{q+1}$ be a decomposition of $\bar{g}$ in $B$ into prime elements. Then by (3.7) and the above argument we have that the logarithmic derivatives $\bar{g}_{i}^{-1} \bar{D}\left(\bar{g}_{i}\right) \in B \quad\left(\right.$ and hence $\overline{\mathscr{L}}$ ) and they generate $\overline{\mathscr{L}}$. Note $\sum_{i=1}^{s+1} \frac{D\left(\bar{g}_{i}\right)}{\bar{g}_{i}}=\frac{D \bar{g}}{\bar{g}}$ $=0$. Therefore $\left\{\bar{g}_{i}^{-1} \bar{D}\left(\bar{g}_{i}\right): 1 \leq i \leq q\right\}$ generate $\overline{\mathscr{L}}$ over $\mathbb{F}_{p}$. We will now show 
that they are $\mathbb{F}_{p}$-independent.

Suppose $e_{i} \in \mathbb{F}_{p}, 1 \leq i \leq q$ are such that $\sum_{i=1}^{q} e_{i} \bar{g}_{i}^{-1} \bar{D}\left(\bar{g}_{i}\right)=0$. By (5.8) we may assume that the representative $g_{i} \in A$ of $\bar{g}_{i} \in B$ is homogeneous $(1 \leq i \leq q$ $+1)$. Let $H=g_{1}^{e_{1}} \cdots g_{q}^{e_{q}}$. Then $\bar{D}(\bar{H})=0$, which implies by (5.1) that $\bar{L}^{p}$ $\subset \bar{L}^{p}(\bar{H}) \subset \bar{L}^{p}(\bar{g})$. If $\bar{H} \in \bar{L}^{p}$ then $e_{i}=0(\bmod p), \quad 1 \leq i \leq q$ and we're done. Otherwise $\bar{L}^{p}(\bar{H})=\bar{L}^{p}(\bar{g})$ which implies there exists $\alpha_{i} \in A(0 \leq i \leq q)$ such that $\bar{\alpha}_{p}^{p} \bar{H}=\sum_{i=0}^{p-1} \bar{\alpha}_{i}^{p} \bar{g}^{i}$. Since $H, g$ are homogeneous elements and $P$ a homogeneous ideal we may assume that the $\alpha_{j}$ are homogeneous polynomials as well. Since $\operatorname{deg}\left(\alpha_{i}^{p} g^{i}\right)=i(\operatorname{deg}(g))(\bmod p)$ and $\operatorname{deg}(g) \neq 0(\bmod p)$, it follows $\bar{\alpha}_{p}^{p} \bar{H}$ $=\bar{\alpha}_{i}^{p} \bar{g}^{i}$ for some $i=0, \ldots, p-1$. This implies that if $i \neq 0 \bar{g}_{q+1} \in B^{p}$, which contradicts (5.1). Thus $i=0$ and $\bar{H} \in \bar{L}^{p}$.

\section{References}

[1] Blass, P., Joyce, D. and J. Lang, The divisor classes of the surface $z^{p}=G(x, y)$, a programmable problem, J. Alg., 101 (1986), 1.

[2] Blass, P. and J. Lang, Zariski Surfaces and Differential Equations in Characteristic $p>0$, Marcel Decker, New York, 1987.

[3] - , A method to computing the kernel of a map of divisor classes of local rings in characteristic $p \neq 0$, Mich. Math. Journal, 35 (1988).

[4] Fossum, R., The Divisor Class Group of a Krull Domain, Springer-Verlag, New York, 1973.

[5] Grant, A. and J. Lang, Applications of the fundamental group and purely inseparable descent to the study of curves on Zariski surfaces, to appear in the Journal of Algebra.

[6] Griffiths and J. Harris, Principles of Algebraic Geometry, Wiley, New York, 1978.

[7] Hartshorne, R., Algebraic Geometry, Springer-Verlag, New York, 1977.

[8] Lang, J., The divisor class group of the surface $z^{p^{m}}=G(x, y)$ over fields of characteristic $p$ $>0, \quad J$. Alg. 2 (1983), 84.

[9] - Generic Zariski Surfaces, submitted.

[10] Matsumura, H., Commutative Ring Theory, Cambridge University Press, Cambridge-New York, 1989.

[11] Samuel, P., Lectures on Unique Factorization Domains, Tata Lecture Notes, 1964. 
